\title{
Power quality monitoring using an integrated Fourier linear combiner and fuzzy expert system
}

\author{
P.K. Dash ${ }^{\mathrm{a}, *}$, R.K. Jena ${ }^{\mathrm{a}}$, M.M.A. Salama ${ }^{\mathrm{b}}$ \\ ${ }^{a}$ Centre for Intelligent Systems, Regional Engineering College, Rourkela 769008, India \\ ${ }^{\mathrm{b}}$ Department of Electrical Engineering, University of Waterloo, Waterloo, Ontario, Canada
}

\begin{abstract}
The paper presents a new approach for the classification of transient disturbance waveforms in a power system by using a Fourier linear combiner and a fuzzy expert system. The measured voltage or current waveforms at a distribution bus are passed through a Fourier linear combiner block to provide peak or root mean square (RMS) amplitude and phase of the fundamental component at every sampling instant. The peak or RMS amplitude and computed slope of the waveforms are then passed on to a diagnostic module that computes the truth value of the signal combination and determines the class to which the waveform belongs. Computer simulated tests are carried out using EMTP programs to obtain the disturbance waveform classification with the help of a new hybrid approach which is much simpler than the recently postulated neural network and wavelet based techniques. The classification is found to be robust and yields accurate results in most cases with the least amount of computational burden.
\end{abstract}

Keywords: Fourier linear combiner; Fuzzy expert system; Disturbance waveform classification

\section{Introduction}

Power supply quality issues and the resulting problems are the consequences of the increasing use of solid state switching devices, non-linear and power electronically switched loads, unbalanced power systems, lighting controls, computer and data processing equipments as well as industrial plant rectifiers and inverters. These electronic type loads cause quasi-static harmonic dynamic voltage distortions, inrush, pulse type current phenomena with excessive harmonics and high distortion. Further cyclic and acyclic loads with temporal variations, or sudden starting of large induction motors can cause low frequency voltage waveform modulation producing a major power quality problem termed as voltage flicker.

Power quality problems can cause system equipment malfunction, voltage flickers, computer data loss and memory malfunction of sensitive loads such as computer, PLC (Programmable Logic Controller) controls, protection and relaying equipment as well as erratic operation of electronic controls.

Power supply quality monitoring involves the estimation of voltage or current peak or root mean square (RMS) values from the measured voltage or current samples and the classification of the waveforms. In addition, the power quality monitoring device displays the captured waveforms when certain thresholds are exceeded. These waveforms exhibit certain distinguishing characteristics and can be classified to belong to a certain waveform class like voltage sag, voltage swell, outage, voltage impulse, or normal etc. The most important characteristic of the classification system is the accuracy and robustness in the presence of noise and harmonics in the data collected from the transmission and distribution networks. Artificial neural networks (ANN) have attracted a great deal of attention because of their pattern recognition capability, parallel computational architecture, associate memory, and the ability to handle noisy data. However, the convergence speed, robustness and accuracy of ANN base methods depend heavily on the choice of the architecture of the network, the weight adaptation algorithm and the amount of noise in the data.

The application of ANN for the classification of low and high impedance faults [1-4], magnetizing inrush [5] and power quality assessment [6] has resulted in significant success. Both multilayered feed-forward and time delay neural networks [7] architectures have been used in classifying transient power quality disturbances like voltage sag, voltage swell, outage, voltage surge etc. The success rate in these classifications varies from 72 to $93 \%$, and the number 


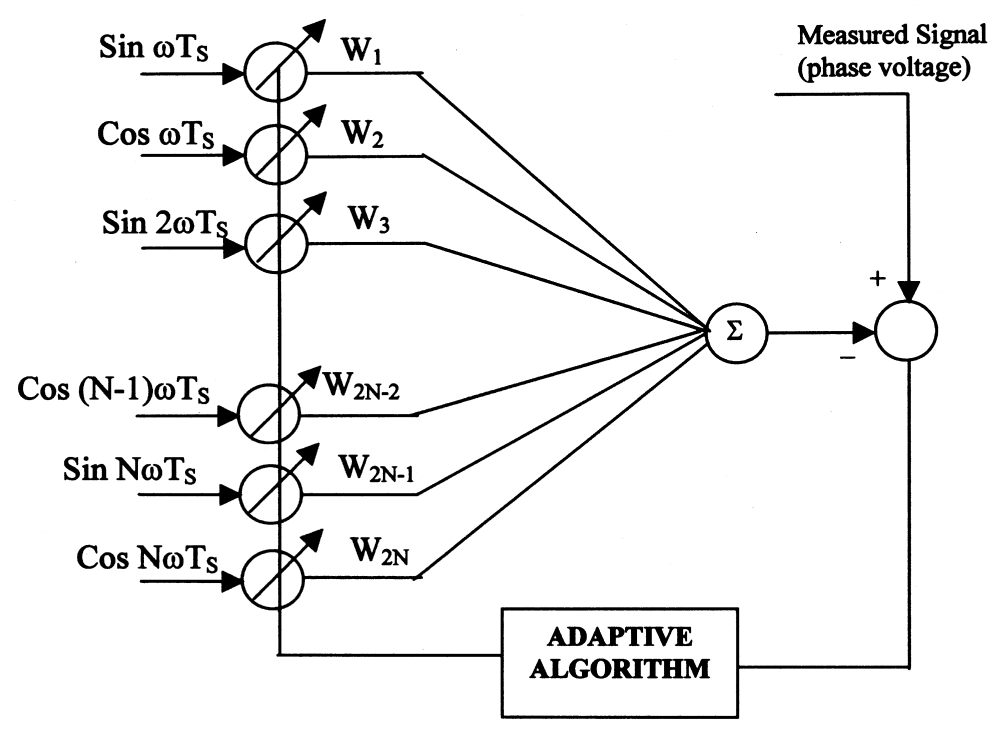

(a) Adaptive LMS Computation Block

(Data Pre Processor)

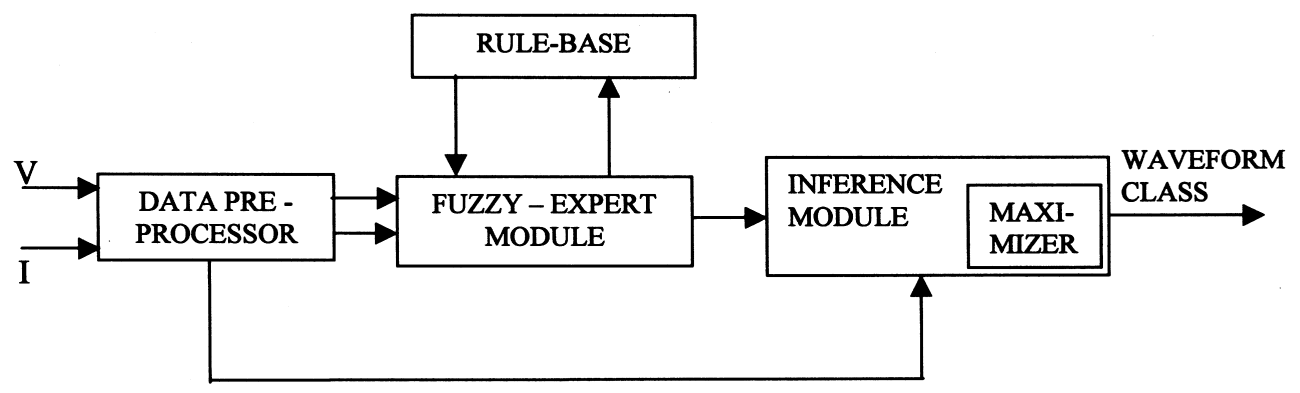

(b) Fuzzy - Expert Module

Fig. 1. Block diagram for power quality monitoring

of training cycles and computational overheads are very large. Another powerful technique presented in the recent time to assess the power quality disturbance type is the wavelet transform [8]. The wavelet transform results in a robust classification at the cost of a large computational overhead as several levels of wavelets in time and frequency frame have to be considered to yield the proper class of the disturbance pattern.

This paper, therefore, presents a new approach using a Fourier linear combiner and a fuzzy rule-based expert system to classify the disturbances. The Fourier linear combiner is used to estimate the phase, amplitude, and rate of change of the voltage waveform of the power system under various operating conditions. The Fourier linear combiner is in the form of an adaline [9], which has an input sequence, and a desired response-signal sequence, and weight parameters. It uses an adaptive algorithm based on a least mean $p$-power error criterion to produce fast convergence and noise rejection unlike the earlier algorithm [9]. The fuzzy expert system [10] uses a rule base to classify the disturbance waveform from the estimated values of amplitude, slope, and distortion factors if any. Several waveforms having swell, sag impulse, outage, harmonic distortion, or frequency excursion etc. embedded in random noise are tested using this new hybrid estimator and fuzzy expert system. The robust and accurate classification of disturbance waveforms validate the efficacy of this new approach. 


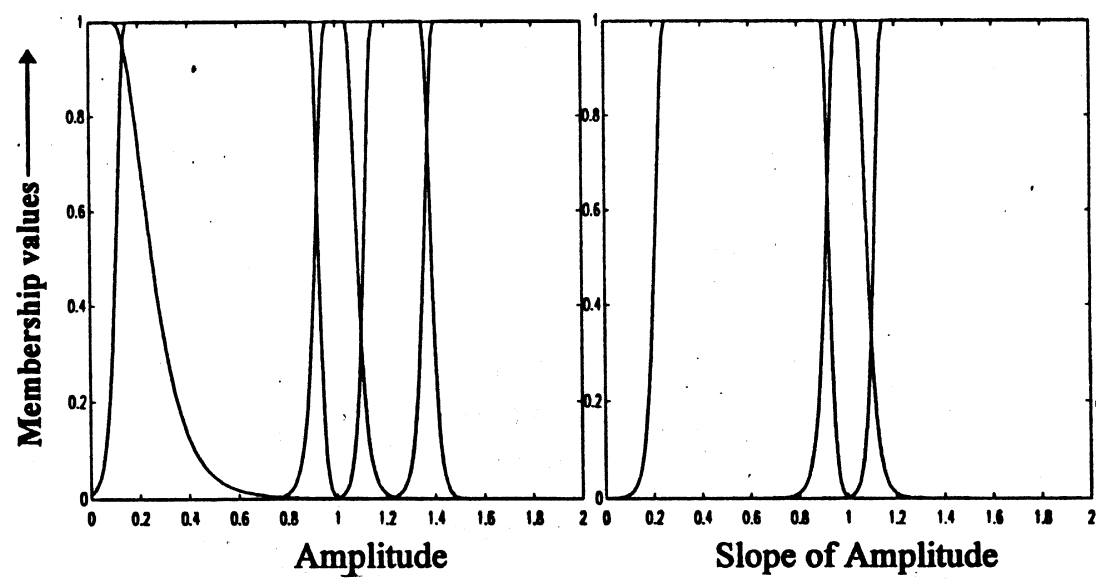

Fig. 2. Membership function for amplitude and slope.

\section{Monitoring and waveform classification strategy}

This section describes the development of a hybrid expert neural system designed to improve the knowledge of the power systems engineer in pursuit of an accurate diagnosis of power system operating problems such as voltage sag, voltage swell, faults, harmonics etc. described below:

- Impulse: The waveform in this class are described as high-frequency transients and occur due to capacitor switching, load start-up and lighting etc.

- Voltage sag: The waveform in this class are characterized by short-term decrease of the voltage magnitude and are measured on a cycle-to-cycle basis. System faults and motor start-ups etc. cause this kind of voltage problem. The majority of voltage sags have a magnitude of around $80 \%$ and a duration of 4-10 cycles.

- Harmonic distortion: Voltage and current signals become distorted due to the harmonic penetration into the power network and voltage and current THDs exceed $5 \%$, which is quite significant. This class of distortion is due to the non-linear loads and adjustable speed drives etc.

- Voltage swell: These waveforms are characterized by short-term increase in line voltage caused primarily by over-excitation, load unbalancing and capacitive loads.

- Outage: An outage is an absence of usable power at some point of the power network, and the waveforms that can be characterized as such fall into this class. These are caused by system faults and opening of circuit breakers.

After choosing the transient disturbance waveforms to be taken up for classification, the next step is to select suitable input features and the method to extract them. Any successful classification would depend on the ability to accurately extract the relevant features from the captured waveform samples in the presence of noise and harmonics. Also the diagnostic module should be easy to implement in real-time without much computational overhead and should yield accurate classifications.
The approach chosen for this purpose consists of a Fourier linear combiner, a preprocessor and a fuzzy diagnostic module. Fig. 1 shows how these modules are interrelated. The raw data in an actual system is to be captured by using a signal conditioner, a data acquisition interface and an analog-to-digital (A/D) conversion kit installed in a PC. This data comprises voltage and current waveforms of a disturbed power system. A Fourier linear combiner module with an adaptive LMS algorithm is then used to estimate the amplitude, phase and THD of the captured waveforms.

\section{Fourier linear combiner}

The voltage or current signal of a power network is expressed in the discrete form such as

$y(k)=s(k)+v(k)=\sum_{i=1}^{N}\left(a_{i} \cos \omega_{i} k+b_{i} \sin \omega_{i} k\right)+v(k)(1)$

where $\omega_{i}$ is the frequency of the $i$ th component and in this case $\omega_{i}$ (the fundamental frequency of the power system signal) is known a priori and $N$ is the order of the harmonics in the signal. In the above formulation, $v(k)$ is the additive white Gaussian noise with zero mean and variance $\sigma_{v}^{2}$ which has no correlation with the signal $s(k)$. A decaying dc component can also be added to the signal model given in (1).

The coefficients $a_{i}$ and $b_{i}$ of the above signal, corrupted by noise $v(k)$ are obtained by minimizing the error $e(k)$ between the desired signal $y(k)$ and the estimated signal $\hat{y}(k)$

$e(k)=y(k)-\hat{y}(k)$

where

$\hat{y}(k)=\sum_{i=1}^{N}\left(\hat{a}_{i} \cos \omega_{i} k+\hat{b}_{i} \sin \omega_{i} k\right)$ 


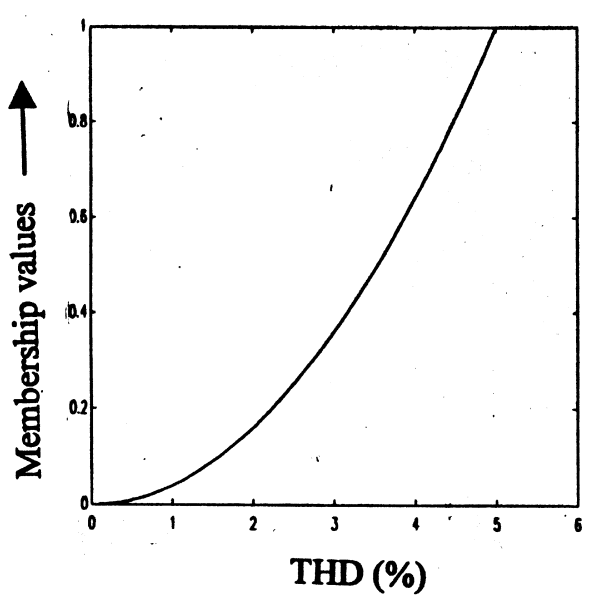

Fig. 3. Membership function for THD.

performance index $J(k)$

$J(k)=E\left[|e(k)|^{p}\right]$.

and $E$ is an expectation operator.

Using the steepest descent algorithm, the parameter $W$ is calculated as

$\hat{W}_{i}(k+1)=\hat{W}_{i}+\frac{\alpha_{i} e^{p-1}(k) x_{i}(k)}{\lambda+x_{i}^{T}(k) x_{i}}$

$i=1,2, \ldots, N$ for even $p$ and

$\hat{W}_{i}(k+1)=\hat{W}_{i}+\frac{\alpha_{i} \operatorname{sign}(e(k)) e^{p-1}(k) x_{i}(k)}{\lambda+x_{i}^{T}(k) x_{i}}$

$i=1,2, \ldots, N$ for odd $p$, where

$x_{i}(k)=\left[\cos \omega_{i}(k) \sin \omega_{i}(k)\right]^{\mathrm{T}}$

$\hat{W}_{i}(k)=\left[\hat{a}_{i}(k) \hat{b}_{i}(k)\right]^{\mathrm{T}}$

and $\alpha_{i}$ is the step size of parameter for the $i$ th frequency component and $\operatorname{sgn}($.$) is the sign function.$

For the value of $p$ greater than 5, the simulations exhibit performance deterioration of the algorithm for tracking power system sinusoids corrupted with noise. Also for $p=$ 1 and 2, the performance of the standard LMS algorithm is obtained. For $p$ taking on values 3 or 4 , the algorithm generates significantly better discrete Fourier transform estimates and hence a value of $p=3$ is chosen in this paper. Thus for $p=3$, the parameter $W$ is updated as

$\hat{W}_{i}(k+1)=W_{i}(k)+\alpha_{i} e^{2}(k) \operatorname{sign}\left\{e(k)\left(x_{i}(k)\right)\right\} /\left[\lambda_{i}\right.$

$$
\left.+x_{i}^{T}(k) x_{i}(k)\right] \text {. }
$$

The step size $\alpha_{i}$ will be selected to be large when there is significant divergence between the actual signal and the computed signal. For small divergence, however, the step size $\alpha_{i}$ is selected to be small. A suitable value of $\alpha_{i}$ lies between 0.2 and 1.6. The adaptive variation of the step size $\alpha_{i}$ is given by

$\alpha_{i}(k)=\alpha_{i}(k-1)+\gamma E_{i}^{2}(k)$
$E_{i}(k)=\beta E_{i}(k-1)+(1-\beta) e_{i}(k) \cdot e_{i}(k-1)$.

In general, the power system disturbance waveforms contain odd and even harmonics, and a decaying dc offset during outages. The presence of the decaying dc offset makes it very difficult to track the fundamental component.
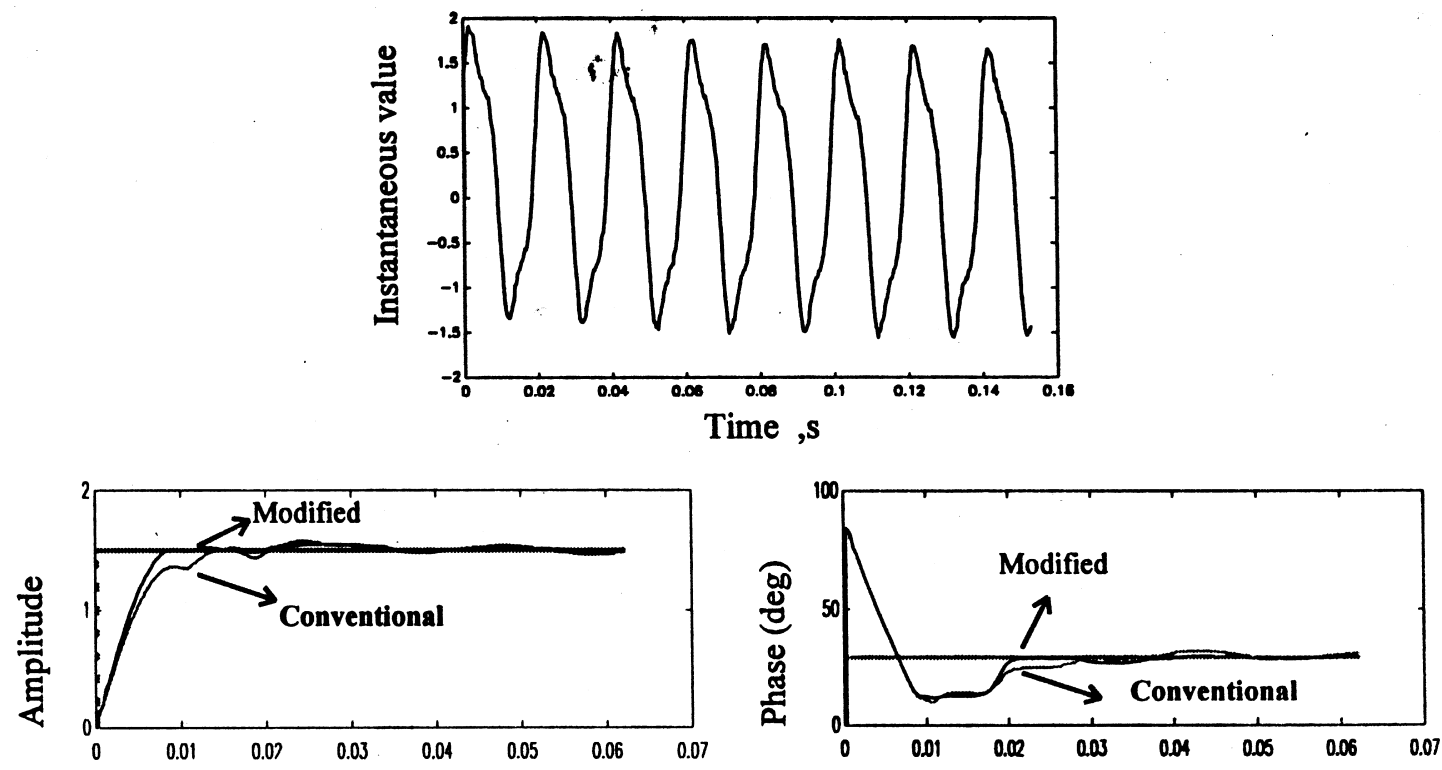

Fig. 4. Comparison of performance of conventional and modified LMS algorithm. 

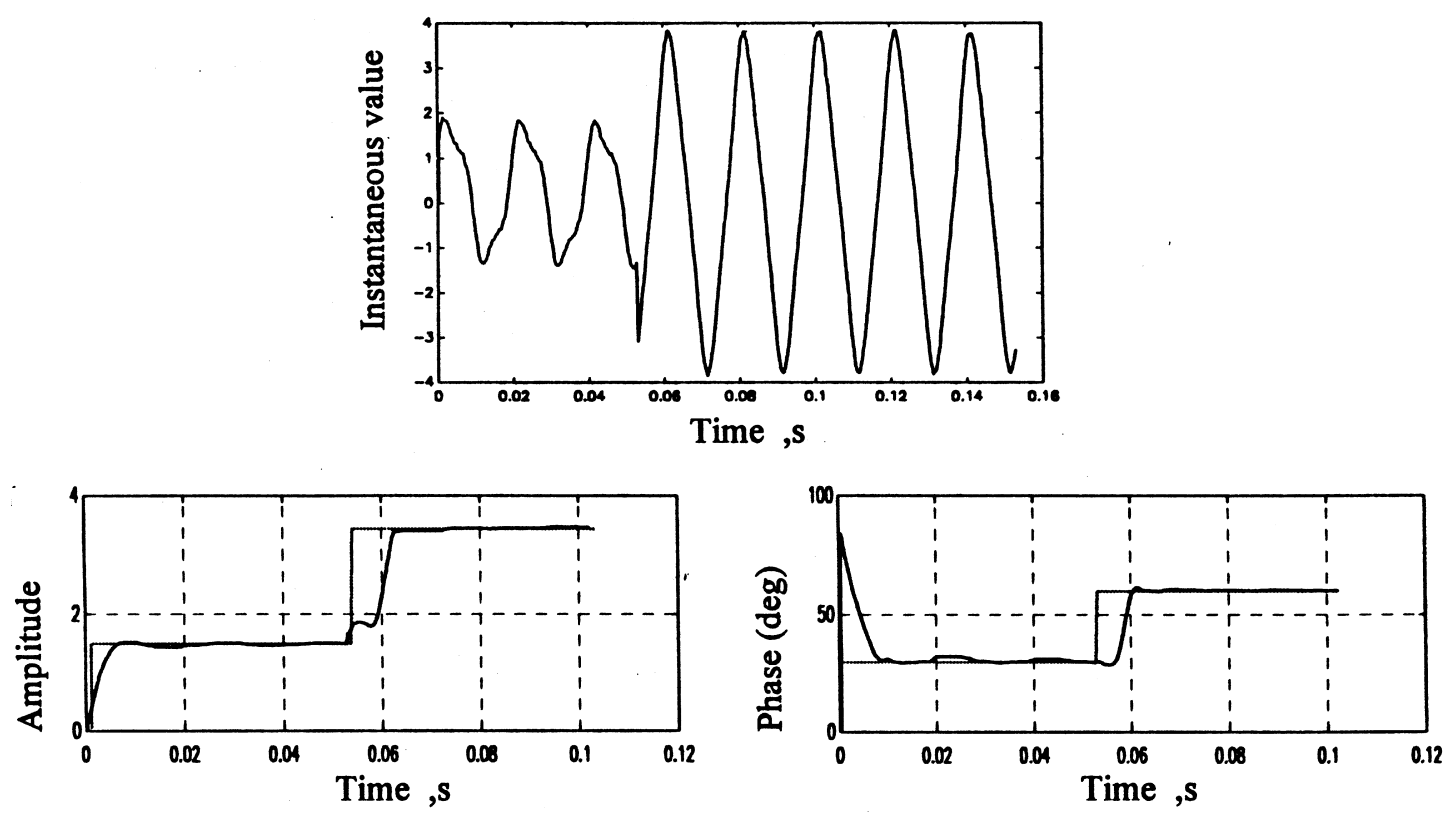

Fig. 5. Amplitude and phase tracking for sudden changes in amplitude and phase of fundamental component in the presence of decaying dc.

The signal embedded with decaying dc can be easily tracked after extracting the dc component as given below.

Let the time varying signal $y(t)$ be represented as

$$
F(t)=I_{0} \mathrm{e}^{-t / \lambda_{\mathrm{dc}}}+I_{m 1} \sin \left(w t+\theta_{1}\right)+I_{m 3} \sin \left(w t+\theta_{3}\right)+\ldots
$$

where $I_{0}$ is the amplitude of dc component and $\lambda_{\mathrm{dc}}$ the decay factor.
The decrement ratio of $I_{0}$ in two consecutive samples is

$$
\beta=I_{0}(m+1) / I_{0}(m)=\mathrm{e}^{-T_{\mathrm{s}} / \lambda_{\mathrm{dc}}}
$$

where $T_{\mathrm{s}}$ is the sampling time.

On the other hand, a small mathematical manipulation shows that

$$
F_{(m)}+F_{(m+N)}=I_{0} \beta_{\mathrm{dc}}^{m}\left(1+\beta_{\mathrm{dc}}^{N}\right)
$$

where $N$ is half of the number of samples per cycle of
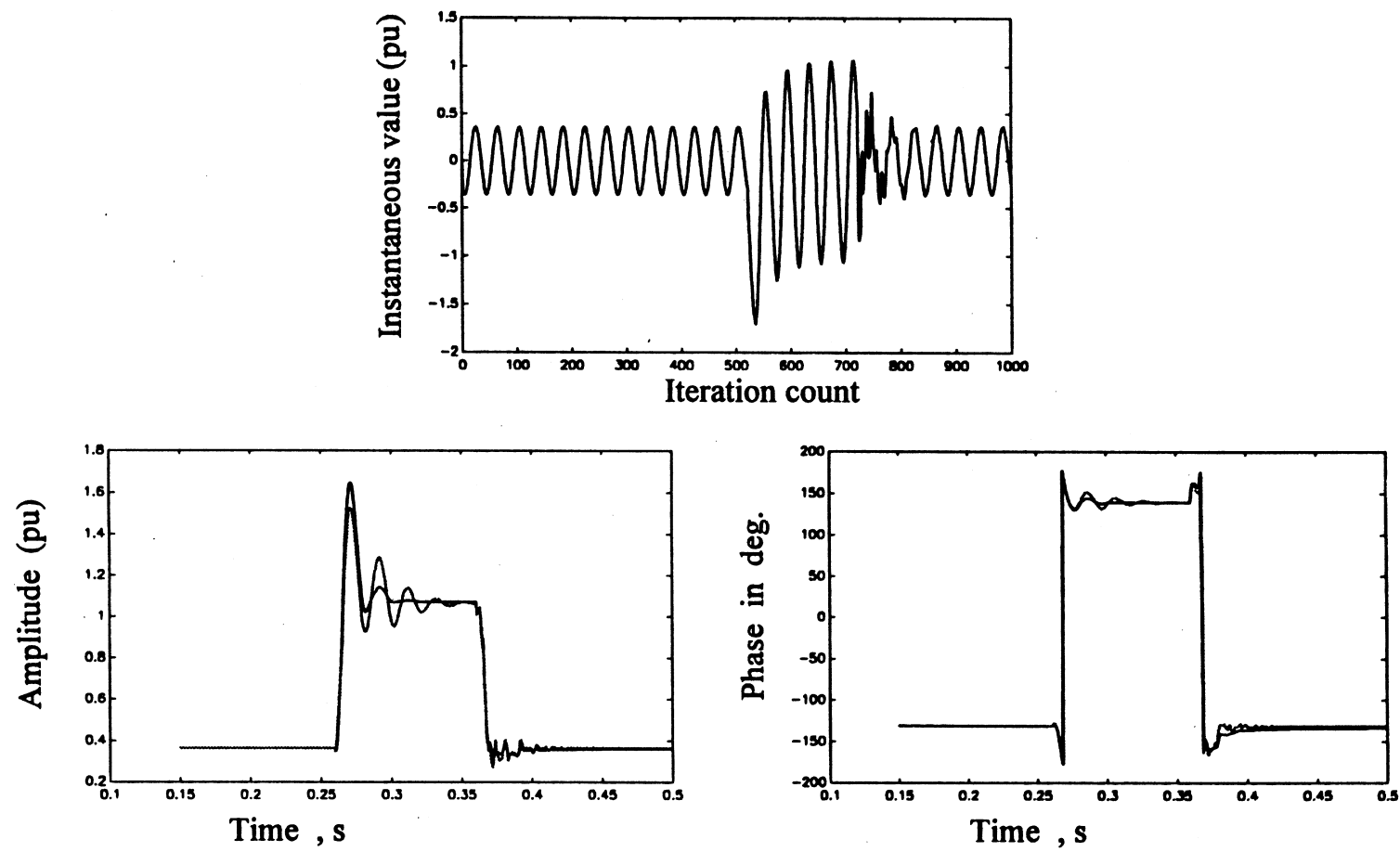

Fig. 6. Comparison of tracking of a fault fundamental current using conventional and new modified LMS algorithm. 

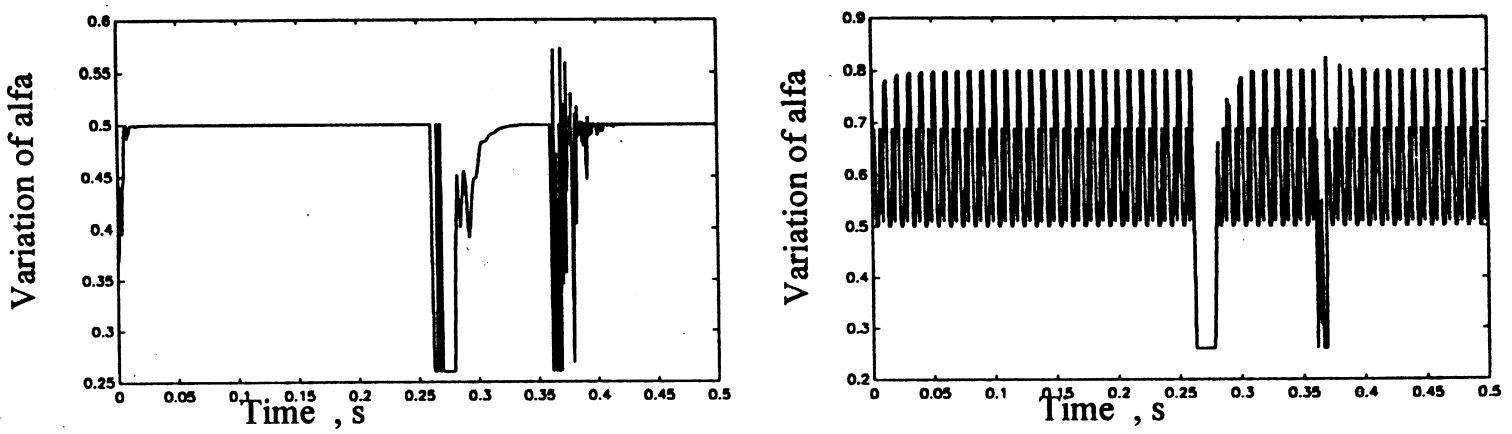

Fig. 7. (i) Variation of alfa in conventional LMS algorithm; and (ii) variation of alfa in modified LMS algorithm.

fundamental waveform and $m$ the present sample $\beta_{\mathrm{dc}}$ and $\lambda_{\mathrm{dc}}$ are calculated as

$\beta_{\mathrm{dc}}=\frac{F_{m+1}+F_{m+N+1}}{F_{m}+F_{m+N}} \quad \lambda_{\mathrm{dc}}=\frac{t_{\mathrm{s}}}{\ln \left(\beta_{\mathrm{dc}}\right)}$

$I_{0}=\left[F_{(m)}+F_{(m+N)}\right] /\left[\beta_{d c}^{m}\left(1+\beta_{d c}^{N}\right)\right]$.

From the above calculation, it is clear that with a minimum of $(N+4) / 2$ samples the dc component can be filtered out.

\section{Fuzzy expert system for classification}

After obtaining the peak or RMS value of the disturbed power system signal, a fuzzy logic based expert systems used to classify the type of disturbance. As the power system data is uncertain and the disturbance classification is a pattern recognition problem, the fuzzy expert system is well suited to this kind of problem. The fuzzy expert system is designed using the knowledge about the disturbances occurring on a distribution bus.

For classifying the power system transient disturbances, three fuzzy sets designated as SN, SZ and SP are chosen for the absolute value of the slope (normalized) or change in the amplitude of the peak value of the waveform. The membership function for computing the absolute value of the slope $S$ is obtained using a bell shaped function as

$\mu(x)=\frac{1}{\left[1+\frac{x-a_{1}}{c}\right]^{b}}$ for $x<a_{1}$

$\mu(x)=1$ for $a_{1}<x<a_{2}$

and

$\mu(x)=\frac{1}{\left[x \frac{-a_{2}}{c}\right]^{b_{2}}}$ for $x>a_{2}$.

In a similar way, five fuzzy sets designated as ALN, ASN, AZ, ASP and ALP are chosen for the peak absolute amplitude $A$ of the waveform. The membership functions are computed using Eq. (14). Fig. 2 shows the membership function for amplitude $A$ and slope $S$ of the disturbance waveform. The normalized slope is calculated as $S(K)=$ $[A(K)-A(K-1)] / S_{\max }, S_{\max }$ is the maximum slope and $k$ the sampling instant. In computing the membership functions, the values of $a_{1}, a_{2}, b_{1}, b_{2}$ and $c$ for the fuzzy set ALN are chosen as $a_{1}=0.0, a_{2}=0.1, b_{1}=2.0, b_{2}=3.0, c=0.3$. For the fuzzy set SN, the above constants are: as $a_{1}=0.25$, $a_{2}=0.9, b_{1}=4.0, b_{2}=8.0, c=0.1$. If the transient disturbance contains harmonics, the total harmonic distortion (THD) can be fuzzified using a membership function of the form

$\mu(\mathrm{THD})=\frac{1}{1+\left(\frac{\mathrm{THD}-5}{50}\right)^{0.2}}$

where THD is expressed in percentage. From the equation, it is quite evident that upto $5 \%$ of THD in a power network is tolerated and beyond 5\%, the membership grade of THD is 1 . Fig. 3 shows the membership function of the total harmonic distortion.

The fuzzified inputs are inferred to a fuzzy rule base, which is used to characterize the relations between fuzzy inputs and fuzzy outputs. In this study, the fuzzy rule base of the fuzzy expert system is fixed as shown below.

Rule 1 If $\mathrm{A}(\mathrm{K})=\mathrm{ASP}$ and $\mathrm{S}(\mathrm{K})=\mathrm{SP}$ then $\mathrm{W}=$ Swell

Rule 2 If $\mathrm{A}(\mathrm{K})=\mathrm{ALP}$ and $\mathrm{S}(\mathrm{K})=\mathrm{SP}$ then $\mathrm{W}=$ Surge

Rule 3 If $\mathrm{A}(\mathrm{K})=\mathrm{ASN}$ and $\mathrm{S}(\mathrm{K})=\mathrm{SN}$ then $\mathrm{W}=\mathrm{Sag}$

Rule 4 If $\mathrm{A}(\mathrm{K})=\mathrm{AZ}$ and $\mathrm{S}(\mathrm{K})=\mathrm{SZ}$ then $\mathrm{W}=$ Normal

Rule 5 If $\mathrm{A}(\mathrm{K})=\mathrm{ALN}$ and $\mathrm{S}(\mathrm{K})=\mathrm{SN}$ then $\mathrm{W}=$ Surge

Rule 6 If $\mathrm{A}(\mathrm{K})=\mathrm{ASP}$ and $\mathrm{S}(\mathrm{K})=\mathrm{SZ}$ then $\mathrm{W}=$ Swell

Rule 7 If $A(K)=A L N$ and $S(K)=S N$ then $W=$ Surge

Rule 8 If $\mathrm{A}(\mathrm{K})=\mathrm{ALP}$ and $\mathrm{S}(\mathrm{K})=\mathrm{SZ}$ then $\mathrm{W}=\mathrm{Sag}$

Rule 9 If $\mathrm{A}(\mathrm{K})=\mathrm{ALN}$ and $\mathrm{S}(\mathrm{K})=\mathrm{SZ}$ then $\mathrm{W}=$ Outage

Rule 10 If $A(K)=A L N$ and $S(K)=S P$ then $W=$ Outage

In the above, $W$ stands for the disturbance waveform.

Although the power system disturbance belongs to five categories like voltage sag, swell, outage, surge and normal, the harmonic distortion can be present in each of them. 

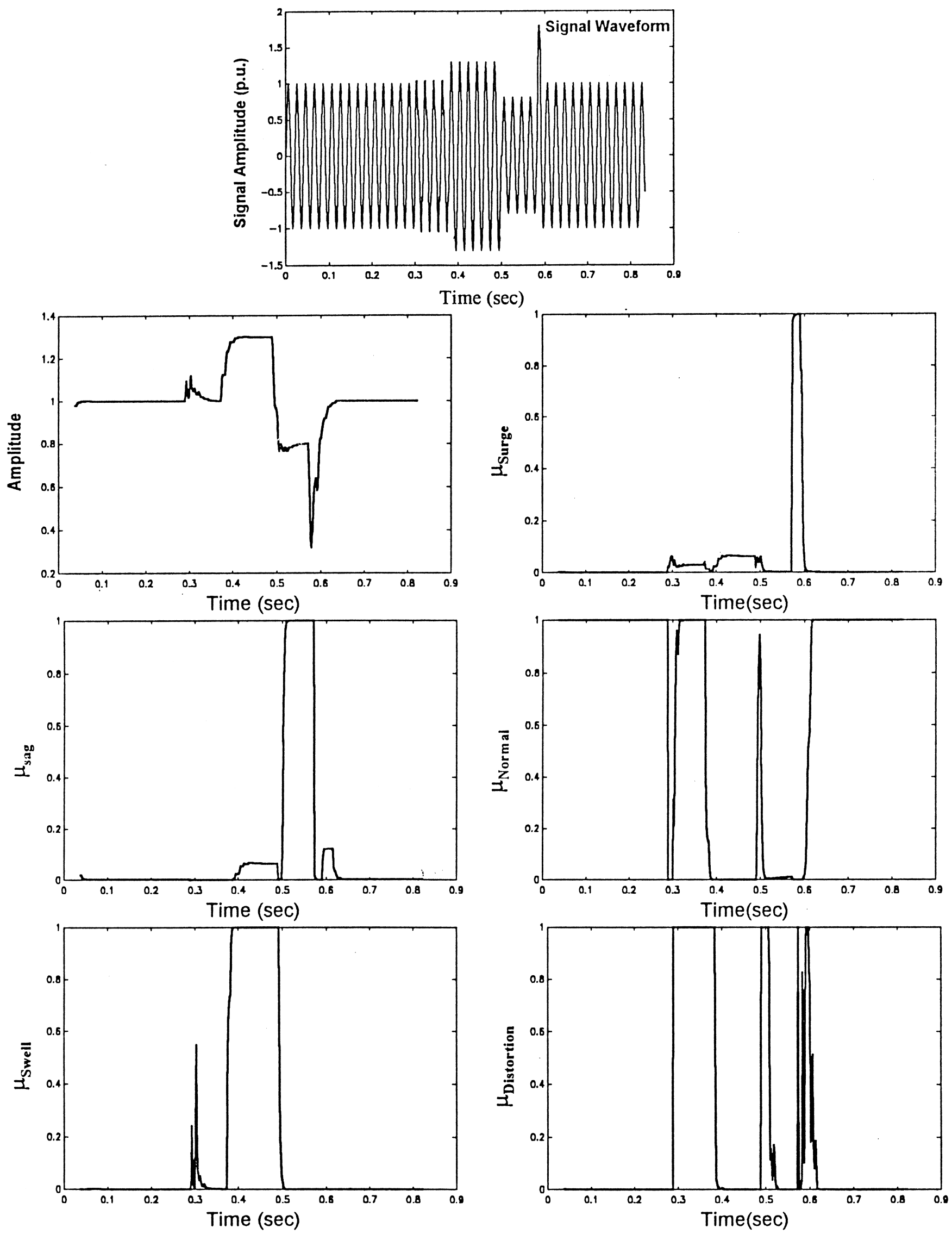

Fig. 8. Output of the fuzzy-module for the composite signal (noise-free case). 


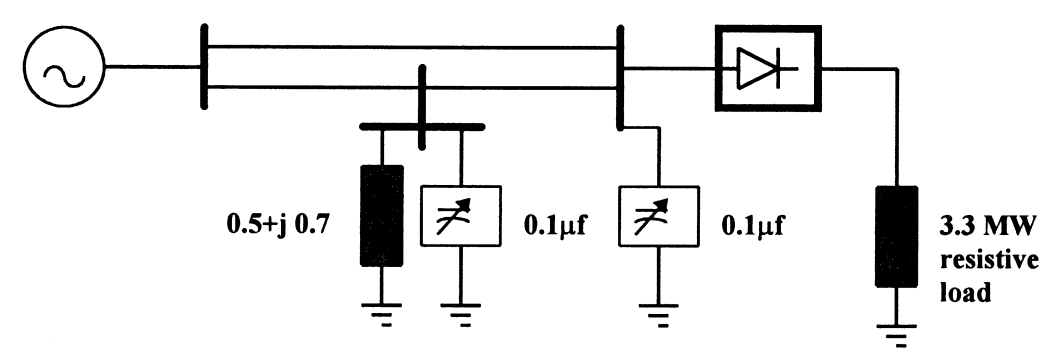

Fig. 9. System configuration of the model used for testing the fuzzy disturbance classifier.

Hence, two more rules are added to the fuzzy rule base to classify the distortion as

Rule 11 If $\mu$ (THD) $<1$ then $\mathrm{W}=$ Normal

Rule 12 If $\mu$ (THD) $>1$ then $\mathrm{W}=$ Distorted

Unlike conventional fuzzy inferencing procedures, the inferencing is done using the maximum product rule of fuzzy inference. Assuming the above five categories of the disturbance outlined above, the output $\left(\mu_{0}\right)$ from the fuzzy expert system module is obtained as

$\mu_{0}(k)=\mu_{1} V \mu_{2} V \mu_{3} V \mu_{4} V \mu_{5}=\max \left(\mu_{1}, \mu_{2}, \mu_{3}, \mu_{4}, \mu_{5}\right)$

where $V$ stands for OR operator and $\mu_{1}, \mu_{2}, \mu_{3}, \mu_{4}, \mu_{5}$ are the firing strengths of the rules for each category of disturbances.

An uncertainty index $\lambda$ is incorporated to the computation process to get the final value of the output $\mu_{0}$, and which is designated as $\mu_{0 \mathrm{~F}} . \mu_{0 \mathrm{~F}}$ is related to $\mu_{0}$ as

$\mu_{0 \mathrm{~F}}(k)=\lambda \mu_{0}(k)+(1-\lambda) \mu_{0}(k-1)$.

The uncertainty index $\lambda$ is used to compensate the discrepancy between the actual value and the observed value. This is due to the time lag introduced by the transducer used for measurements of power system disturbance waveforms. Further, the severity problem is realised when the magnitude of the voltage phaser changes is accompanied by a change in the phase angle as is observed in the case of

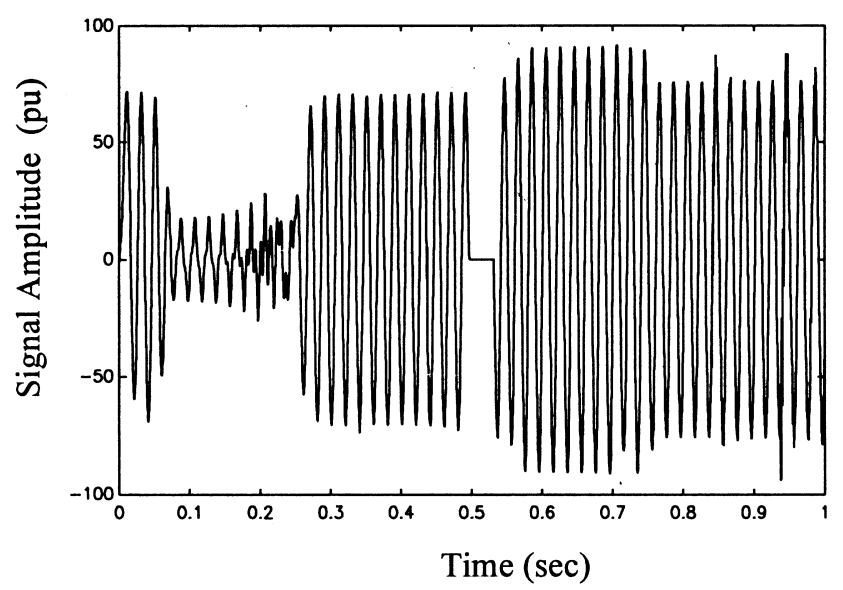

Fig. 10. Converter bus voltage (phase- $a$ ). transformer switching and starting of large induction motors.

\section{Simulation results}

Computer simulated waveforms for various transient disturbances of a power system are generated using MATLAB. The sampling rate of 16 based on a $50 \mathrm{~Hz}$ waveform is used for testing the effectiveness of the new algorithm in classifying disturbance waveforms. A SGN function is used for updating the weight vector of the neural estimator which is initialised using a set of random weights. The value of initial parameter is chosen and the limits $\alpha_{\max }$ and $\alpha_{\min }$ are maintained as 1.2 and 0.6 , respectively. The value of $\beta$ and $\gamma$ are chosen as $\beta=0.8$ and $\gamma=.01$.

\subsection{Monitoring of disturbance waveforms}

For testing the efficacy of the new algorithm, the following power system signal corrupted with noise and decaying dc component is used for simulation:

$$
\begin{aligned}
y(t)= & 1.5 \sin (w t+\pi / 6)+0.3 \sin (3 w t+\pi / 10) \\
& +.02 \sin 5 w t+0.3 \exp (-12 t)+.03 \operatorname{rand}(t) \\
& \text { for } t<.05312 \mathrm{~s}
\end{aligned}
$$

and

$$
\begin{aligned}
y(t)= & 3.5 \sin (w t+\pi / 3)+.3 \sin (3 w t+\pi / 10)+.0 \sin (5 w t \\
& +\pi / 4)+.03 \operatorname{rand}(t)
\end{aligned}
$$

for $t>.05312 s$.

From the above description of signals it can be observed that the signal has a sudden jump at time $t$ greater than. $05 \mathrm{~s}$ approximately. Also the decaying dc component is present in the signal at time $t<.0535$. The signal is corrupted by a random white noise of variance $\sigma=.03$ and zero mean. The tracking efficiencies of the standard LMS algorithm and the least mean $p$-power error criterion for the above mentioned signal are shown in Fig. 4. In case of the conventional LMS algorithm, the decaying dc component is filtered out using the principle described in Section 4. From the results, it can be seen that the $p$-power error criterion yields a better 

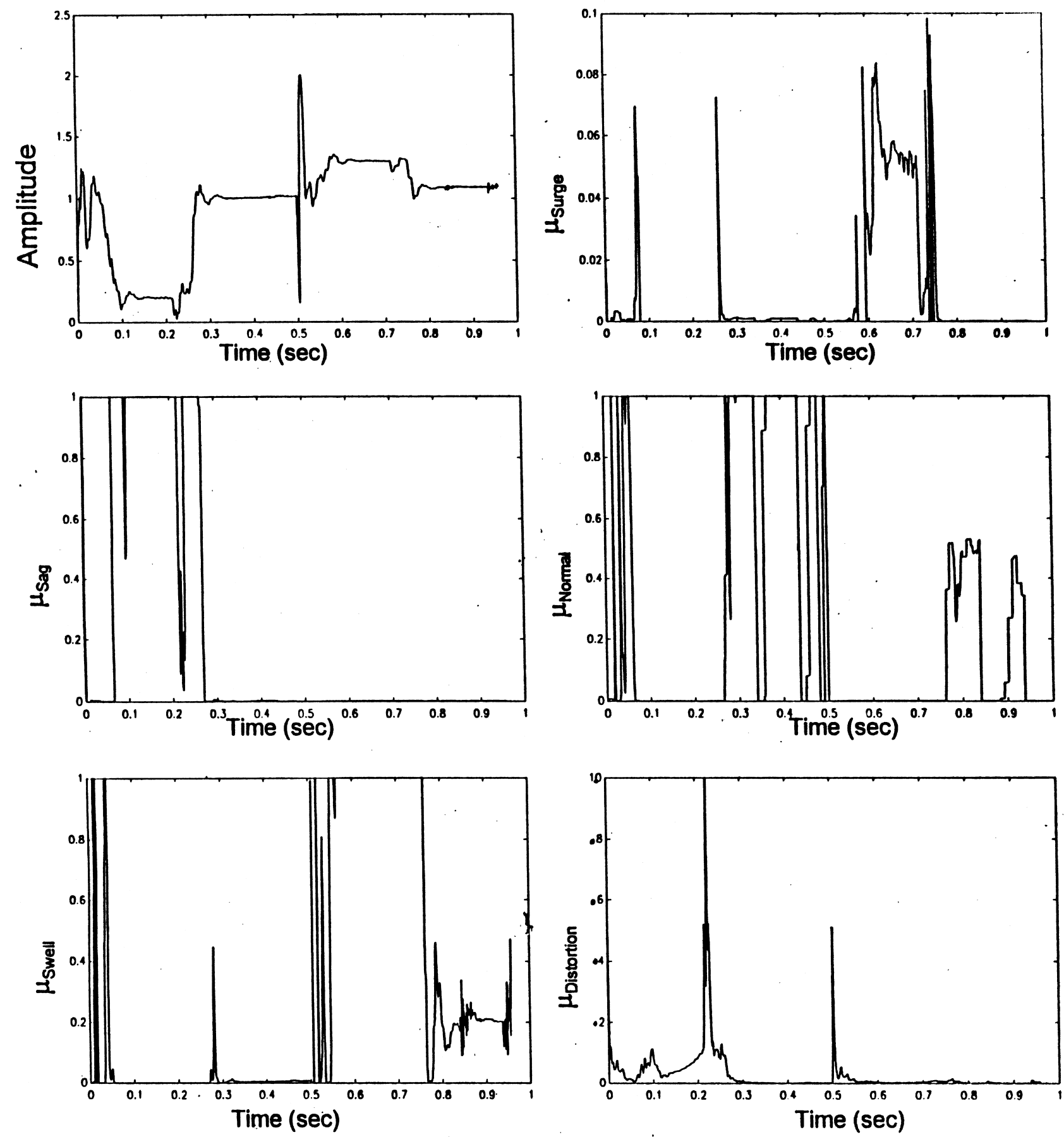

Fig. 11. Output of fuzzy-expert module for the test waveform.

tracking capability of the fundamental component of the power system disturbance signal when dc offset is present. The tracking time is less than a cycle based on the fundamental frequency of the signal. This new algorithm does not require any filtering technique as needed for the conventional LMS approach in the presence of random noise.

Further, the new algorithm performs extremely well (Fig. 5) during sudden changes in the amplitude and phase angle of the fundamental component, which is the one required for monitoring and classification. Kalman filter is also used to track the signal component in the presence of decaying dc and random noise and the result is not as good as the one obtained using the new approach. The results for the Kalman filter are not presented in the paper.

Another interesting case of practical value is the tracking of the fundamental component of the power system disturbance waveform during a fault.

An EMTP software is used to simulate a synchronous generator feeding a transmission line subjected to a single-line-to-ground fault at a certain distance from the 
sending end. The fault current waveform is shown in Fig. 6 . This figure also presents the amplitude and phase angle of the fundamental component of the faulted current waveform. The generator frequency also varied during the fault, which was estimated by another frequency estimation algorithm presented in Ref. [10].

From the results presented in the figure, it can be seen that the mean $p$-power error criterion produces an excellent convergence to the true signal value in the presence of noise, decaying dc components, harmonics and frequency excursion. If the frequency excursion is very small, the tracking time remains within a cycle.

The conventional LMS algorithm shows large oscillations due to frequency changes and the presence of dc offset and noise. The variations of $\mu$ for both the conventional LMS and the new approach with mean $p$-power error criterion are shown in Fig. 7.

\subsection{Classification of disturbance waveforms}

Fig. 8 shows the category of the simulated waveforms like sag or swell and the corresponding output from the Fourier linear combiner and the integrated fuzzy and Fourier linear combiner diagnostic systems. The training of the monitoring system for different class of waveform is essential for tuning the parameters of the various modules. From the figure, it is observed that each category of waveform is successfully classified as the output from the model, shows a $100 \%$ truth value of the particular class that suddenly rises from 0 to $100 \%$ in most cases in comparison to the normal waveform.

After ascertaining the efficacy of the proposed fuzzy expert system in classifying the simulated disturbances, a practical case is taken for a detailed study. Fig. 9 shows a generator supplying a power network which comprises a short-transmission line section and resistive and constant impedance loads. The resistive load consumes 3.3 MW of power and is supplied from a rectifier connected to the load bus as shown in Fig. 9. The initial starting current of the rectifier is $18 \mathrm{~A}$ and is started at $t=0.043 \mathrm{~s}$. An outage at the generator end is initiated at $t=0.49 \mathrm{~s}$ and persists for 2 cycles based on $50 \mathrm{~Hz}$ waveform. Variable static capacitors are installed at the load bus to improve the load power factor as shown in the figure. The converter voltage waveform (one phase only) is presented in Fig. 10 using an EMTP software package. Fig. 11 presents the classification results showing clearly the type of disturbance class (voltage sag or voltage swell etc.) from changes in membership grades. For a particular type of disturbance, the membership grade will become unity and for other non-occuring types, membership grades will be very small.

\section{Conclusion}

The paper presents a new approach to the assessment of power quality using an integrated Fourier linear combiner and fuzzy decision support system. The tracking capability of the Fourier linear combiner is enhanced with an adaptive LMS algorithm using a new $p$-power error criterion. A fuzzy logic based reasoning process is used to classify the power quality disturbance waveforms from the computed amplitude and slope of the waveforms obtained from the Fourier linear combiner. The newly modified LMS algorithm tracks the fundamental component of the signal corrupted with noise within a time period less than one cycle. The paper also presents the variation of the learning rate using the new $p$-power error criterion. The fuzzy expert system used for the classification power network disturbances like voltage sag, voltage swell, outage, voltage surge etc. is very simple to implement in a real time in comparision to the recently proposed ANN and wavelet based approaches, which are computationally very involved. Several computational test results are also presented in the paper to show the improved performance of the integrated Fourier linear combiner and fuzzy logic approach.

\section{References}

[1] Lubkeman DL, Fallon CD, Girgis AA. Unsupervised learning strategies for the detection and classification of transient phenomena on electric power distribution systems, Proceedings of First International Forum on Applications of Neural Networks to Power Systems, IEEE 91 THO 374-9, 1991, pp. 107-111.

[2] Kim CJ, Russell BD. Classification of faults and switching methodology. IEEE Transactions on Power Delivery 1989;4(3):1631-1637.

[3] Ebron S, Lubkeman D, White M. Neural network power distribution feeders. IEEE Transactions on Power Delivery 1990;5(2):905-914.

[4] Sultan AF, Swift GW, Fedirchu DJ. Detection of high impedance arcing faults using a multi-layer perceptron. IEEE Transactions on Power Delivery 1992;7(4):1871-1877.

[5] Perez LG, Flechsig AJ, Meador JL, Obradovic Z. Training an artificial neural network to discriminate between magnetizing inrush and internal faults, IEEE Winter Power Meeting, 1993, paper no.WM037-2, PWRD.

[6] Daniels R. Power quality monitoring using neural networks, Proceedings of the First International Forum on Application of Neural Networks to Power Systems, IEEE 91 THO 374-9, 1991, pp. 195-197.

[7] Ghosh AK, Lubkeman DL. The classification of power system disturbance waveforms using a neural network approach. IEEE Transactions on Power Delivery 1995;10(1):109-115.

[8] Dash PK, Swain DP, Liew AC, Rahman S. An adaptive linear combiner for on-line tracking of power system harmonics, 1996, papers IEEE 96 WM 181-8 PWRS.

[9] Dash PK, Liew AC, Rahman S. Fuzzy neural network and fuzzy expert system for load forecasting. Proceedings IEE (London), Generation, Transmission and Distribution 1996;143(1):106-115.

[10] Dash PK, Swain DP, Routray A, Liew AC. An adaptive neural network approach for the estimation of power system frequency. Electric Power Research 1997;41:203-210. 